\title{
Wolf Huetteroth · Joachim Schachtner \\ Standard three-dimensional glomeruli of the Manduca sexta antennal lobe: a tool to study both developmental and adult neuronal plasticity
}

\begin{abstract}
The metamorphosing antennal lobe (AL) of the sphinx moth Manduca sexta serves as an established model system for studying neuronal development. To improve our understanding of mechanisms involved in neuronal plasticity, we have analyzed the size, shape, and localization of ten identified glomeruli at three different time points during development and in the adult, viz., (1) 13 days after pupal eclosion (P13), which reflects a time when the basic glomerular map has formed, (2) immediately after adult eclosion (A0), which represents a time when the newly formed glomeruli are uninfluenced by external odors, and (3) 4 days after adult eclosion (A4), which reflects a time when the animals have been exposed to surrounding odors. Our data from normally developing ALs of male M. sexta from P13 to A0 revealed an increase in size of all examined glomeruli of between $40 \%$ and $130 \%$, with the strongest increases occurring in two of the three sex-specific glomeruli (cumulus, toroid). From A0 to $\mathrm{A} 4$, the cumulus and toroid increased significantly when correlated to $\mathrm{AL}$ volume, whereas the other glomeruli reached the sizes gained after A0. This study was based on antibody staining against the ubiquitous synaptic vesicle protein synaptotagmin, confocal laser scan microscopy, and the three-dimensional (3D) analysis tool AMIRA. Tissue permeability and therefore reliability of the staining quality was enhanced by using formalin/methanol fixation. The standard 3D glomeruli introduced in this study can now be used as basic tools for further examination of neuronal plasticity at the level of the identified neuropil structures, viz., the glomeruli of the AL of M. sexta.
\end{abstract}

Keywords 3D reconstruction - AMIRA - Brain · Glomerular map $\cdot$ Olfactory system $\cdot$ Manduca sexta males, (Insecta)

W. Huetteroth $\cdot$ J. Schachtner $(\triangle)$

Fachbereich Biologie, Tierphysiologie, Philipps Universität,

35043 Marburg, Germany

e mail: schachtj@staff.uni marburg.de

\section{Introduction}

The two antennal lobes of the adult sphinx moth Manduca sexta are the first relay stations for olfactory information processing in the brain. The neuropil of the antennal lobe (AL) is organized in globular structures, the so-called glomeruli $(n=63 \pm 1)$, which are arranged in a sphere around a central coarse neuropil (Rospars and Hildebrand 2000). This glomerular assembly seems to be a conserved morphological principle among brain regions involved in the initial central processing of olfactory information, since it is, with variable numbers of glomeruli, common in insects and vertebrates (for reviews, see Hildebrand and Shepherd 1997; Strausfeld and Hildebrand 1999; Eisthen 2002).

The AL arises during approximately 3 weeks of hormonally controlled pupal development (regarded as pupal stages P0 to P20; Truman 1996; Schachtner et al. 2004b). During this time, the whole brain undergoes reorganization, and a small larval antennal center develops into the adult AL (Oland and Tolbert 1996). All neurons of the AL have been born by P3 (Hildebrand et al. 1997). Beginning with $\mathrm{P} 4$, the olfactory receptor neurons (ORNs) enter the $\mathrm{AL}$ and induce, in association with antennal glial cells, the formation of glomeruli during the subsequent stages (for a review, see Tolbert et al. 2004). After a short delay, mainly between P7/8 and P12, the interaction of ingrowing ORNs, glial cells, local (LNs) and projection (PNs) neurons leads to increased synaptogenesis in a distoproximally directed wave and to the stabilization of the glomeruli (Oland et al. 1990; Tolbert and Sirianni 1990; Dubuque et al. 2001). After P12, the glomeruli increase in size, probably because of the increasing neurite diameters, but the main wave of synaptogenesis seems to be complete (Tolbert et al. 1983; Tolbert 1989; for reviews, see Oland and Tolbert 1996; Tolbert et al. 2004). Nevertheless, the synaptic connections in the glomeruli are thought to undergo further maturation and refinement until eclosion (Tolbert et al. 1983; Dubuque et al. 2001).

Several authors have outlined the advantages of a 3D map not only of vertebrate brains (Ruffins et al. 2002; also reviewed in Van Essen et al. 2002), but also of insect brain 
structures (Rohlfing et al. 2004; Zöckler et al. 2001; Chiang et al. 2001; Rein et al. 1999, 2002; Haddad et al. 2004), especially in the AL (Drosophila melanogaster: Laissue et al. 1999; Apis mellifera: Flanagan and Mercer 1989; Galizia et al. 1999; Blaberus craniifer: Chambille and Rospars 1981; Mamestra brassicae: Rospars 1983; Helicoverpa assulta and Heliothis virescens: Berg et al. 2002; Spodoptera littoralis: Sadek et al. 2002; Agrotis ipsilon: Greiner et al. 2004; Cotesia glomerata and rubecula: Smid et al. 2003). The identification and 3D representation of a set of "ordinary" glomeruli in an atlas allows for their repeatedly reliable identification irrespective of the original data (Galizia et al. 1999). Rospars and Hildebrand $(1992,2000)$ reconstructed ALs of adult $M$. sexta based on paraffin sections, but, although they provided the first map of $M$. sexta AL glomeruli, their work supplied only restricted information about glomerular volumes or shape. Because of the immense improvements in confocal laser scan microscopy (CLSM), computer power, and 3D reconstruction software during last few years, 3D maps have been constructed of various insect brains. However, a reconstruction of the AL of $M$. sexta by these methods has been absent until now, mainly because of its large size.

We have pursued two main aims in this study: (1) the quantification of the increase in glomerular size during the second half of pupal development and during the first few days after adult eclosion, and (2), for future studies, the establishment of an anatomical and volumetric standard of ten glomeruli of P13 pupae, freshly eclosed adults (A0), and 4-day old adults (A4). Within the scope of this study, we have found significant glomerular size increases in two of three sex-specific glomeruli in adult $M$. sexta. We have also found evidence for a higher variability in malespecific glomerular shape between individuals than has previously been reported (Rospars and Hildebrand 1992, 2000). Some of this study has been published before in abstract form (Huetteroth and Schachtner 2003).

\section{Materials and methods}

Animals

Moths (Manduca sexta; Lepidoptera: Sphingidae) were kept in walk-in environmental chambers at $26^{\circ} \mathrm{C}$ under a long-day photoperiod (L:D=17:7) and were fed on an artificial diet as described by Bell and Joachim (1978). Under this regime, pupal development from the day of pupal ecdysis (P0) until adult eclosion (A0) took approximately 20 days, each day corresponding to a pupal stage (P0-P20). Adult life was staged accordingly (A0-A4). Adult animals were supplied with water ad libitum; they were allowed to smell but not to reach the opposite sex. Pupae were staged according to criteria described in Jindra et al. (1997) and Schwartz and Truman (1983). Stages of other staging systems (Tolbert et al. 1983) used in cited studies were adapted accordingly. Only male moths were used.

\section{Immunocytochemistry}

As a primary antibody, we used a monoclonal anti-synaptotagmin antibody from mouse $(1: 2,000$, kindly provided from Kaushiki Menon, Caltech, USA). Specificity controls for the anti-synaptotagmin antibody are described in Dubuque et al. (2001). As a secondary antibody, Cy3-coupled goat anti-mouse antibody (Jackson Immuno Research, Westgrove, Pa., USA) was used at a dilution of 1:300. After dissection in cold saline (Weevers 1966) or phosphatebuffered saline (PBS, $0.01 \mathrm{M}$ ), brains of various developmental stages of $M$. sexta were fixed in methanol containing $10 \%$ formalin for $50-100 \mathrm{~min}$ at room temperature and then rinsed in PBS for $1 \mathrm{~h}$ at room temperature. Because of the large brain size, two slabs containing the ALs were cut out of each brain (see also Figs. 2; 3a, A, $\mathrm{A}^{\prime \prime}$ ). These slabs were preincubated overnight at $4^{\circ} \mathrm{C}$ in $5 \%$ normal goat serum (NGS; Jackson Immuno Research) in $0.1 \mathrm{M}$ TRIS$\mathrm{HCl} / 0.3 \mathrm{M} \mathrm{NaCl}$ (SST) with $0.5 \%$ Triton X-100 (SST-TX 0.5 , Sigma-Aldrich Chemie). The primary antibody was then diluted in SST-TX 0.5 with $1 \%$ NGS and incubated with the slabs for 5-6 days at $4^{\circ} \mathrm{C}$. The brain slabs were rinsed 12 times over $6 \mathrm{~h}$ in SST-TX 0.1 at room temperature. Secondary antibody (in SST-TX $0.5 / 1 \%$ NGS) was applied for 2 days at $4^{\circ} \mathrm{C}$, followed by rinsing in SST-TX 0.1 at room temperature for $4 \mathrm{~h}$. Slabs were dehydrated in an ascending alcohol series (30\%-100\%, 5 min each), cleared in methyl salicylate (Merck, Gernsheim, Germany), and subsequently mounted in Permount (FisherScientific, Pittsburgh, Pa., USA). We used spacers to diminish squeezing of the preparations and two coverslips to allow scanning of the material from both sides.

\section{CLSM image acquisition and processing}

Fluorescently labeled slabs were scanned at $1024 \times 1024$ pixel resolution by using a $20 \times$ objective (HC PL APO $20 \times / 0.70$ Imm Corr CS; Leica, Bensheim, Germany) on a confocal laser scan microscope (Leica TCS SP2). No correction for $z$-axis refractive index mismatch was necessary, since the immersion oil, mounting medium, and tissue had refractive indices $\mathrm{N}_{\mathrm{d}}{ }^{20}$ of approximately $1.5(\mathrm{Gahm}$ and Witte 1986). Because of to the limited working distance of the objective $(170 \mu \mathrm{m})$ and the size of the slabs (approximately $300 \mu \mathrm{m}$ ), we had to scan the material from two sides to obtain images of the whole preparation. This resulted in two image stacks. Further processing of images (reduction to $512 \times 512$ pixels and conversion to an 8 -bit gray-scale) was performed by using Adobe Photoshop 6 . Corresponding optical slices in the overlapping part of both image stacks were found by using the "Align Slices" tool of AMIRA 3.0 and 3.1 (Indeed-Visual Concepts, Berlin, Germany). Redundant slices of the stack were abolished. This led to image stacks of ALs and adjacent protocerebrum with a resolution of $1.4 \mu \mathrm{m} \times 1.4 \mu \mathrm{m} \times 1.01$ $\mu \mathrm{m} /$ voxel and an overall size of about $720 \mu \mathrm{m} \times 720$ $\mu \mathrm{m} \times 272-325 \mu \mathrm{m}$. 
Glomerular structures in the AL were labeled as described previously by using the AMIRA "brush" tool, "interpolate" tool, and "warp" tool (Galizia et al. 1999; Smid et al. 2003). To determine boundaries between two adjacent glomeruli more precisely, the "4viewer" mode was employed, which allows assessment of the glomerular extent in the $\mathrm{XY}$ plane, $\mathrm{XZ}$ plane, and $\mathrm{YZ}$ plane of each respective image stack (Fig. 1). The volumes of labeled glomeruli were read out with the tool "tissue statistics". The data were imported and further processed with Microsoft Excel XP.

Because of the variable orientation of the preparation, the CLSM sectioning of the AL could not always be achieved in orthogonal frontal planes corresponding to anatomical axes. This problem was solved by reslicing the CLSM stacks with the AMIRA "ObliqueSlice" tool until a corresponding plane consistent with previous data was detected.

Differences in absolute (measured glomerulus volumes) and relative (glomerulus volumes correlated to AL volume) glomerular volumes between $\mathrm{P} 13$ and $\mathrm{A} 0$ and between $\mathrm{A} 0$ and A4 were examined with the Mann-Whitney $U$ test, since we observed no normal distribution of data within groups. We used the Friedman analysis of variance and Kendall coefficient of concordance test to determine whether there was constancy in glomerular size within the various stages. Significances were calculated with the Statistica"99 edition.

\section{Results}

Overall, we acquired image stacks of 37 ALs obtained from 26 male moths. Stages included P12 (3 ALs from 3 animals), P13 (6 ALs from 5 animals), A0 (16 ALs from 9 animals), A1 (4 ALs from 4 animals), and A4 (8 ALs from 5 animals). Most of this study including volumetric comparisons is based on P13, A0, and A4 ALs (see Table 1 for a comprehensive overview). The glomeruli of the macroglomerular complex (MGC), the labial pit organ glomerulus (LPOG), and six identifiable "ordinary" glomeruli were routinely analyzed. For reconstructing the whole set of AL glomeruli, 2 ALs of P12, 4 ALs of P13, 1 AL of A0, and 5 ALs of A4 animals were used (Table 1). The methanol-containing fixation allowed for antibody staining throughout the AL preparations; no labeling
Fig. 1 Synaptotagmin immu nostaining in three single planes of about $1 \mu \mathrm{m}$ thickness of an AL whole mount (male, A4). A One single optical slice from a stack of 282 approximately sagittal slices (XY). B, C The same image stack, viewed in YZ (frontal) and XZ (coronal) planes, respectively. Lines refer to corresponding intersection planes in A C. This figure dis plays the quality of the optical slices taken with a lateral reso lution of $1024 \times 1024$ pixels. Thus, the voxel resolution is 0.7 $\mu \mathrm{m} \times 0.7 \mu \mathrm{m} \times 1.01 \mu \mathrm{m}$. Note the slight compression in the $z$ axis and the consistently good stain ing quality throughout the vol ume of the tissue slab. The alignment edge of the two image stacks can barely be seen (ar rowheads). Bar $200 \mu \mathrm{m}$

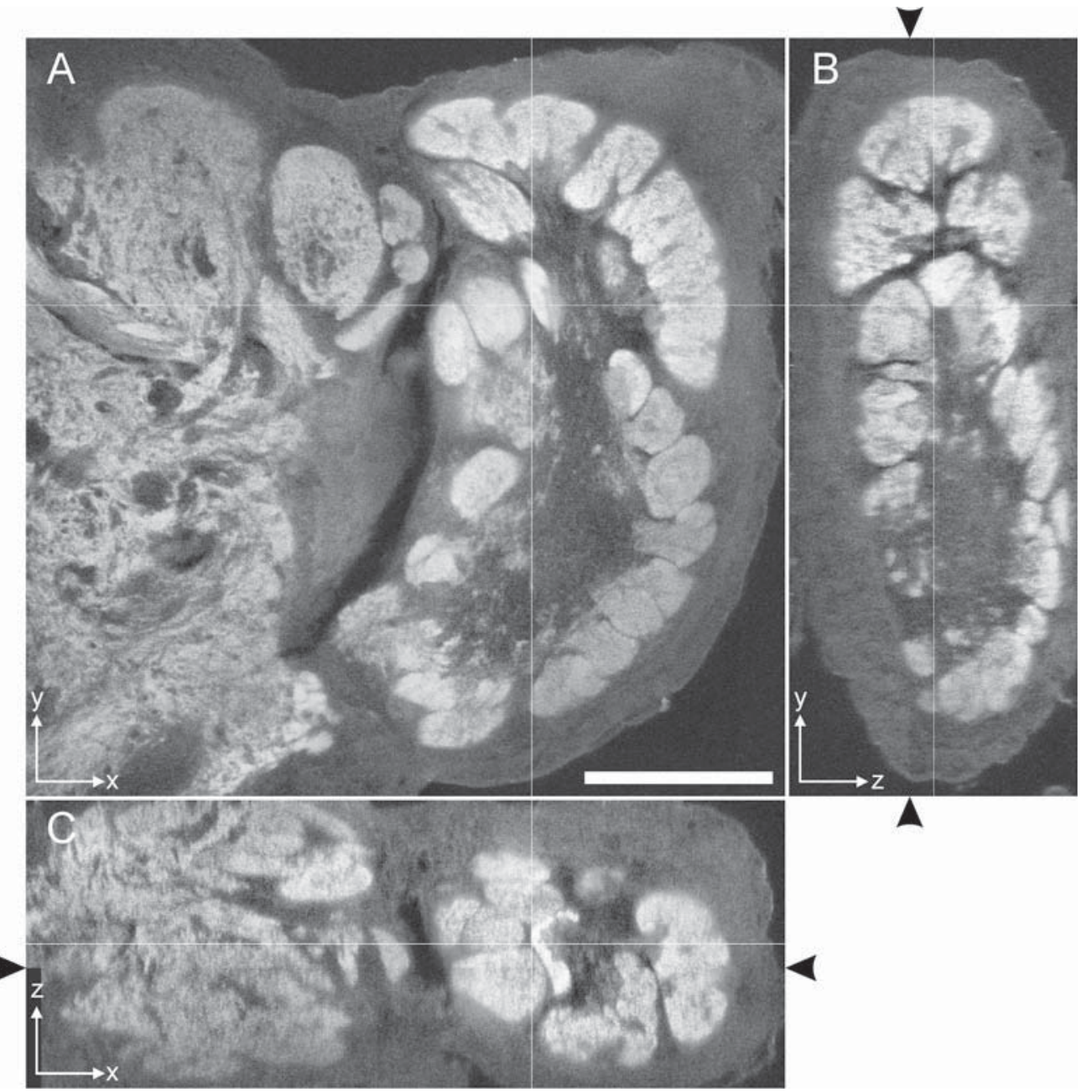


Table 1 Comprehensive over view of antennal lobes (ALs) used in this study. From all

listed ALs, the glomeruli of the MGC, the LPOG, and six

"ordinary" glomeruli were rou

tinely analyzed. Additionally, all glomeruli were labeled from 12 of the 37 ALs. The develop mental stages (the figures in which the corresponding ALs are presented are indicated in brackets), weight of the animal, right $(r)$ or left $(l)$ AL side, number of optical slices per image stack, and additional la bels (All all glomeruli labeled, $T$ ring shaped toroid, $H s$ horse shoe, numbers number of labels assigned to the corresponding glomerulus) are shown.

\begin{tabular}{|c|c|c|c|c|}
\hline Stage & Weight (mg) & Side & Number of slices & Additional labels \\
\hline $\mathrm{P} 12^{\mathrm{a}}$ & 3.78 & $\mathrm{r}$ & 139 & \\
\hline $\mathrm{P} 12^{\mathrm{a}}$ & 4.33 & $\mathrm{r}$ & 238 & All; 2 Hs \\
\hline $\mathrm{P} 12^{\mathrm{a}}$ & 4.80 & $\mathrm{r}$ & 310 & All; T; 2 Hs \\
\hline P13(Fig. 2) & 3.73 & $\mathrm{r}$ & 289 & All \\
\hline P13 & 4.10 & $\mathrm{r}$ & 293 & All; T; 2 Hs \\
\hline P13 & 4.40 & $\mathrm{r}$ & 243 & \\
\hline P13(Fig. 3) & 5.73 & $\mathrm{r}$ & 298 & All; T; 2 Hs \\
\hline $\mathrm{P} 13$ & 5.73 & 1 & 251 & $2 \mathrm{Hs}$ \\
\hline P13 & 5.87 & $\mathrm{r}$ & 254 & All; $\mathrm{T}$ \\
\hline A0(Fig. 2) & 1.82 & $\mathrm{r}$ & 272 & $2 \mathrm{Hs}$ \\
\hline A0 & 1.84 & $\mathrm{r}$ & 277 & 2 Discbase \\
\hline A0 & 1.84 & 1 & 276 & 2 Cumulus; 2 Hs; 2 Club \\
\hline A0 & 2.00 & $\mathrm{r}$ & 289 & $2 \mathrm{Hs}$ \\
\hline A0 & 2.00 & 1 & 287 & $2 \mathrm{Hs}$ \\
\hline $\mathrm{A} 0$ & 2.02 & $\mathrm{r}$ & 268 & 2 Toroid \\
\hline A0 & 2.02 & 1 & 299 & 2 Toroid; 3 Hs \\
\hline A0 & 2.03 & $\mathrm{r}$ & 262 & $\mathrm{~T} ; 2 \mathrm{Hs}$ \\
\hline $\mathrm{A} 0$ & 2.03 & 1 & 298 & $2 \mathrm{Hs}$ \\
\hline A0(Fig. 3) & 2.07 & $\mathrm{r}$ & 299 & All; $4 \mathrm{Hs}$ \\
\hline A0 & 2.07 & 1 & 280 & 2 Cumulus \\
\hline A0 & 2.27 & $\mathrm{r}$ & 298 & $\mathrm{~T} ; 2 \mathrm{Hs}$ \\
\hline A0 & 2.27 & 1 & 304 & $3 \mathrm{Hs}$ \\
\hline $\mathrm{A} 0$ & 2.62 & $\mathrm{r}$ & 279 & $2 \mathrm{Hs}$ \\
\hline $\mathrm{A} 0$ & 2.70 & 1 & 308 & $3 \mathrm{Hs}$ \\
\hline A0 & 2.70 & $\mathrm{r}$ & 326 & \\
\hline $\mathrm{A} 1^{\mathrm{a}}$ & 1.52 & $\mathrm{r}$ & 240 & \\
\hline $\mathrm{A} 1^{\mathrm{a}}$ & 1.63 & $\mathrm{r}$ & 283 & $2 \mathrm{Hs}$ \\
\hline $\mathrm{A} 1^{\mathrm{a}}$ & 1.78 & $\mathrm{r}$ & 275 & $2 \mathrm{Hs}$ \\
\hline $\mathrm{A} 1^{\mathrm{a}}$ & 1.80 & $\mathrm{r}$ & 288 & $\mathrm{~T} ; 2 \mathrm{Hs}$ \\
\hline A4 & 1.20 & $\mathrm{r}$ & 270 & All; T; 2 Hs \\
\hline A4(Fig. 3) & 1.34 & $\mathrm{r}$ & 294 & All; 2 Hs \\
\hline A4(Fig. 1) & 1.37 & $\mathrm{r}$ & 282 & All \\
\hline A4 & 1.37 & 1 & 278 & \\
\hline A4 & 1.38 & $\mathrm{r}$ & 275 & All; T; 3 Hs; 2 Discbase \\
\hline A4 & 1.38 & 1 & 274 & $3 \mathrm{Hs}$ \\
\hline A4 & 1.49 & $\mathrm{r}$ & 292 & All; 2 Hs \\
\hline A4 & 1.49 & 1 & 279 & $2 \mathrm{Hs}$ \\
\hline
\end{tabular}

gradient attributable to limited permeation was detected (Figs. 1, 2).

\section{Organization of AL neuropil}

In addition to the three sex-specific glomeruli of the MGC, we found 59-66 "ordinary" glomeruli of various shapes. Mainly bulb-like shapes were seen, but ellipsoid and sphere-like glomeruli were also observed. Typically, the glomeruli exhibited a more intensely stained apical cortex and a peduncle oriented toward the central coarse neuropil of the AL. With one exception, every single glomerulus contacted the AL core with its peduncle. Only one glomerulus, the "cap" glomerulus, was completely separated from the AL center by another glomerulus, viz., the "base" glomerulus (Figs. 2, 3, blue glomerulus), of which the "cap" might be a subcompartment (Figs. 2, 3, small light orange glomerulus).

The cumulus as the first and most distal glomerulus of the MGC almost always exhibited an elongated cylindrical shape that lay halfway embedded in the furrow of the toroid and oriented in an anteroventral manner (dark blue glomerulus in Figs. 2, 3). In two cases, two labels were assigned to the cumulus (Table 1). The two other sexspecific glomeruli, the toroid and the horseshoe (large orange glomerulus and purple glomeruli, respectively, in Figs. 2, 3), showed higher individual plasticity in shape: the toroid exhibited its name-giving toroidal shape (Homberg et al. 1995) in only 9 of 37 ALs examined; otherwise, it was "U"-shaped, with its opening oriented in an anteroventral fashion. In both ALs in one animal, we found two labels that were assigned to the toroid (Table 1). The horseshoe featured the highest range of shape and volume plastici- 

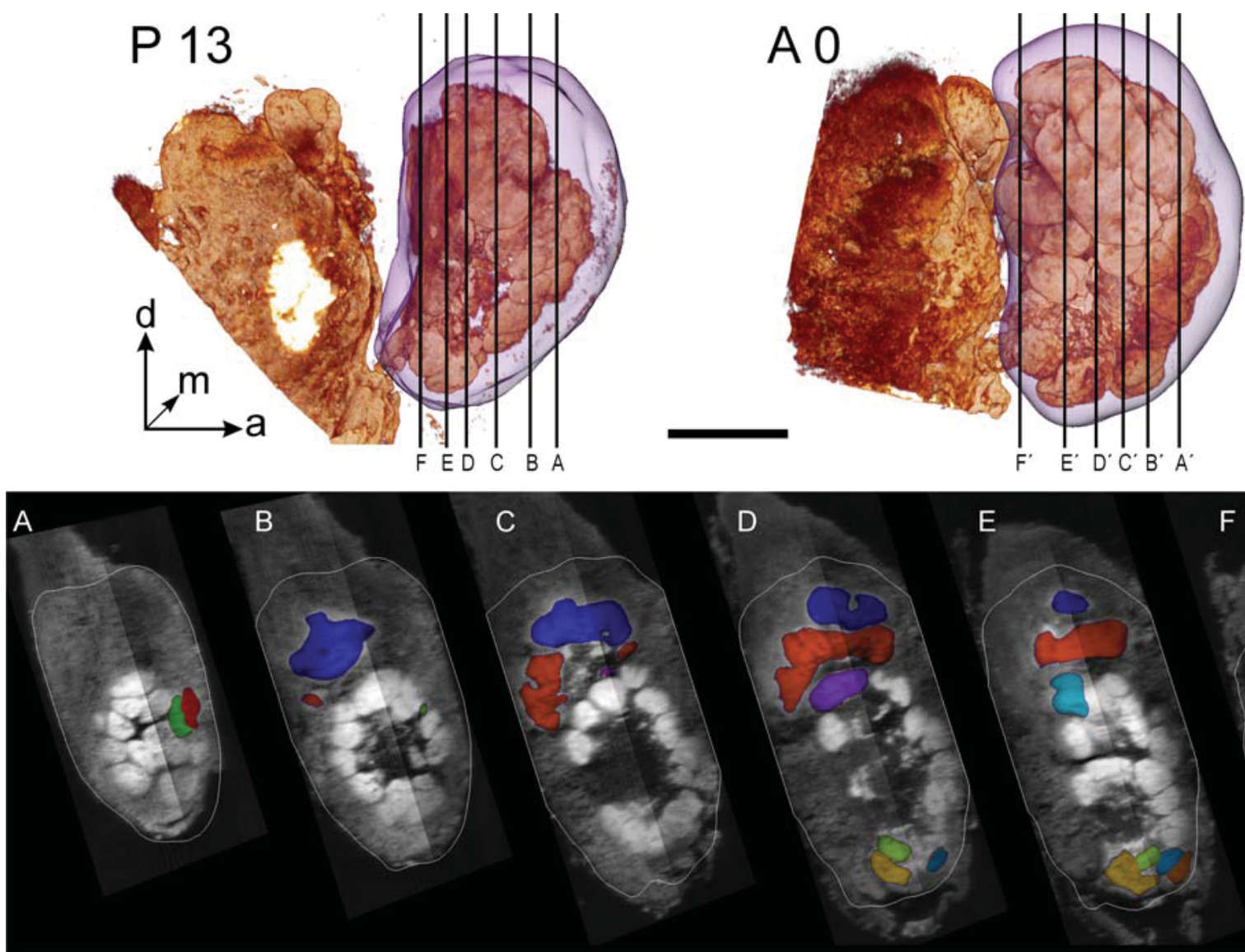

D
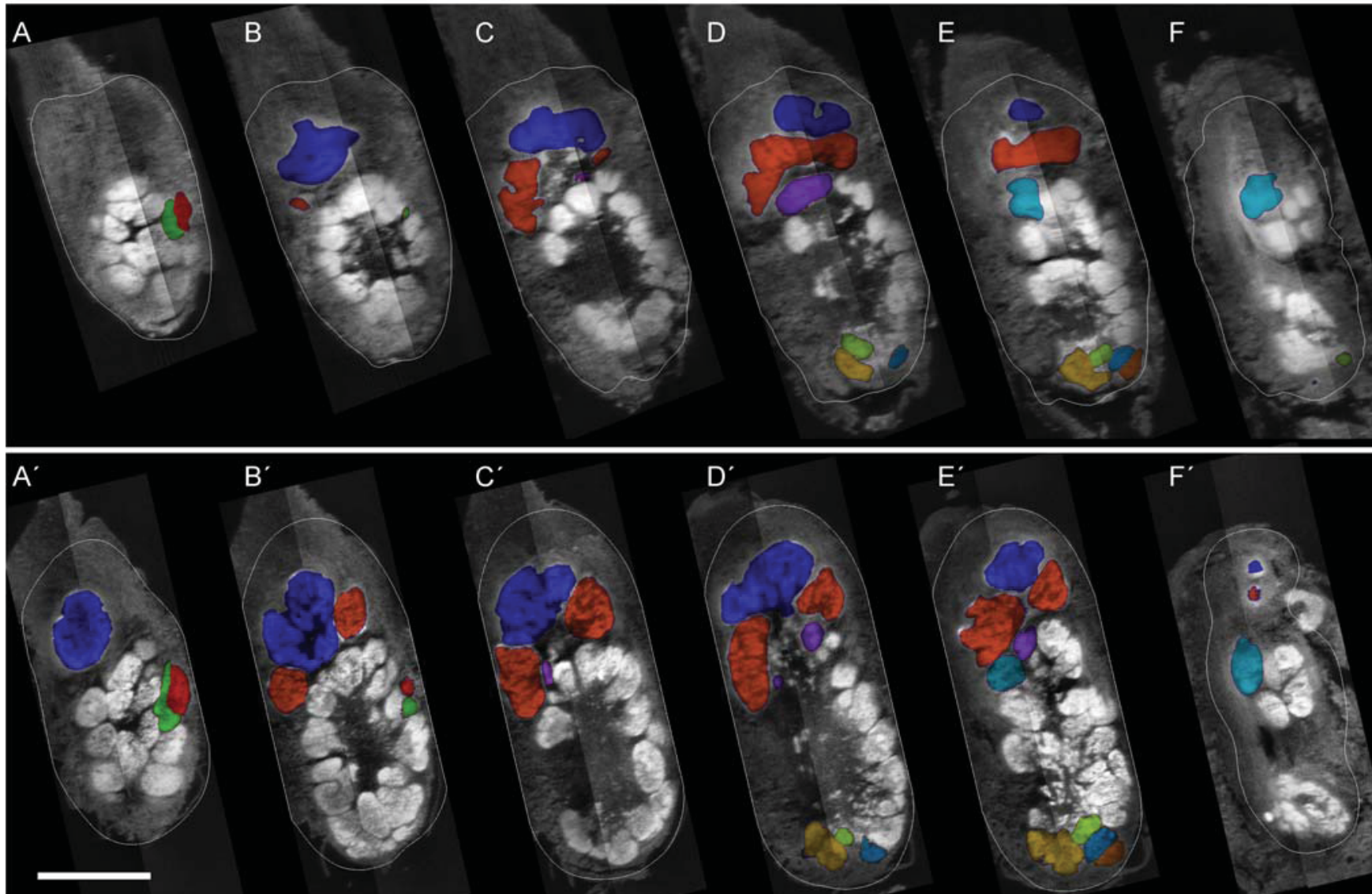

$D^{\prime}$

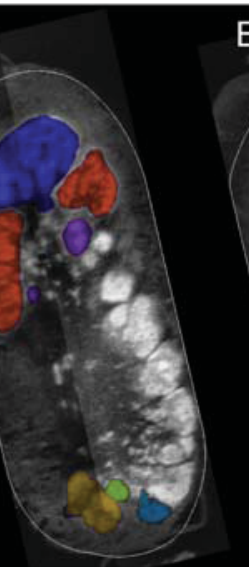

$E^{\prime}$

$\mathrm{F}^{\prime}$

Fig. 2 Lateral view of 3D CLSM reconstructions of right male ALs of P13 and freshly enclosed $M$. sexta (A0). The reconstructed AL volume is visualized transparently. Vertical lines correspond to frontal sections in $\mathbf{A} \mathbf{F}$ (P13) or $\mathbf{A}^{\prime} \mathbf{F}^{\prime}$ (A0), respectively ( $d$ dorsal, $m$ median, $a$ anterior). A F Frontal sections through P13 AL represented by vertical lines in P13 above. Labeled materials are either outlined (AL volume) or color coded (dark blue cumulus, orange toroid, purple horseshoe, light blue G20, dark red disc, green discbase, yellow LPOG, light green club, light orange cap, blue base). $\mathbf{A}^{\prime} \mathbf{F}^{\prime}$ Corresponding sections through A0 AL represented by vertical lines in A0 above. Note the cumulus extending further anteriorly into section $\mathbf{A}^{\prime}$. Identical glomeruli can be found in similar positions during development, although slight individual variations of glom erular location and shape occur. The whole set of labeled frontal sections of both ALs are also available as animated slices online (http://online media.uni marburg.de/biologie/3d brain/). Bar $200 \mu \mathrm{m}$ ty: in 20 of the 37 preparations, the horseshoe comprised two halves; in six cases, we even found that three to four parts had different sizes at the corresponding position of the horseshoe (Table 1). This high variation could explain why the horseshoe neuropil was initially assigned to three different glomeruli (Rospars and Hildebrand 1992, 2000). Normally, its form exhibits the shape and orientation of the toroid, but we found variations ranging from " $U$ "-shapes to kidney shapes. All "ordinary" glomeruli exhibited shape plasticity to a lesser extent (Table 1). 
518
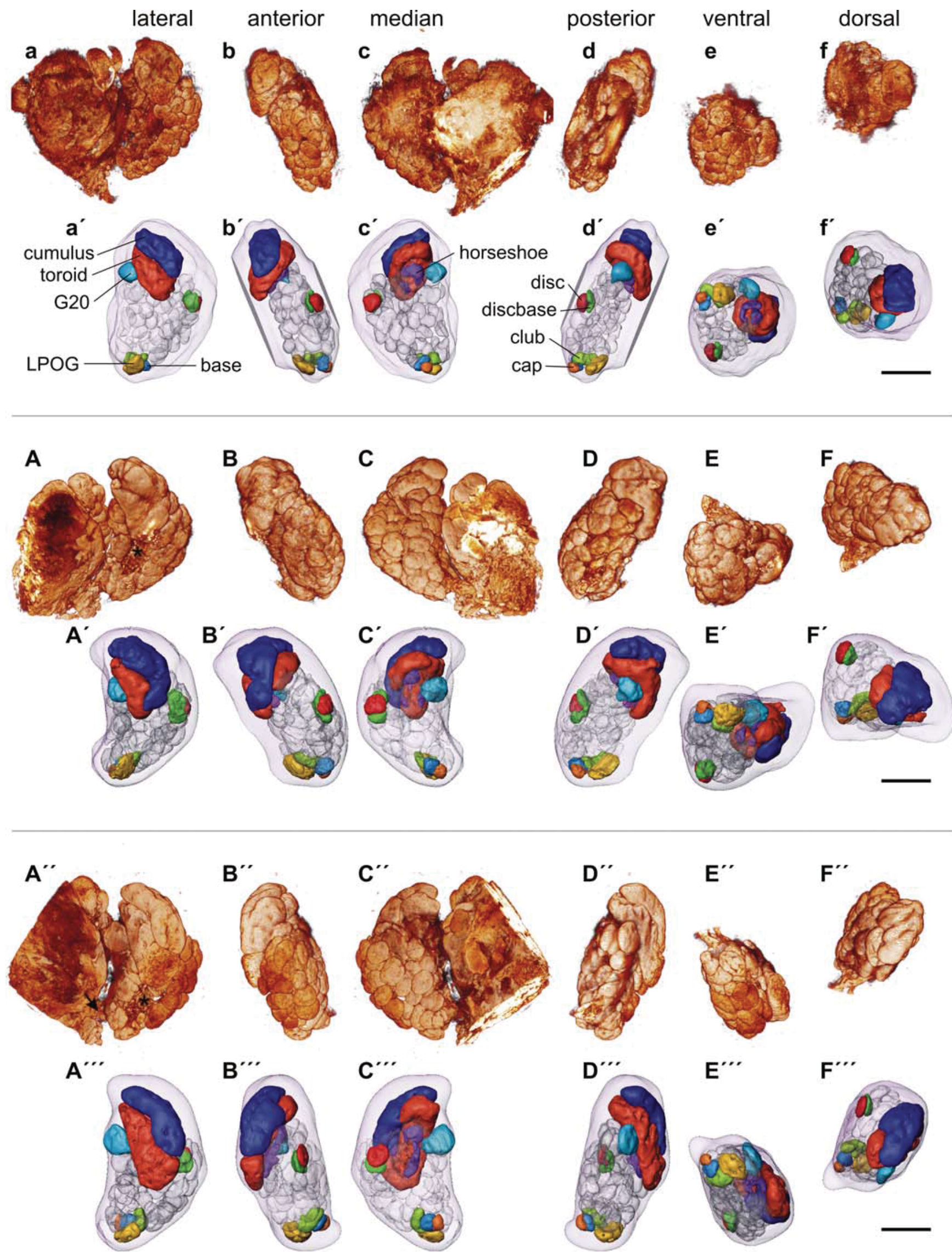
Fig. 3 Surface rendered views of synaptotagmin immunostaining in three representative ALs (a f P13; A F A0; $\mathbf{A}^{\prime \prime} \mathbf{F}^{\prime \prime}$ A4) with corresponding reconstructed glomerulus volumes $\left(\mathbf{a}^{\prime} \mathbf{f}^{\prime} \mathrm{P} 13 ; \mathbf{A}^{\prime} \mathbf{F}^{\prime}\right.$ $\left.\mathrm{A} 0 ; \mathbf{A}^{\prime \prime \prime} \mathbf{F}^{\prime \prime \prime} \mathrm{A} 4\right)$. In the lateral and median view (first and third columns), additional protocerebral staining is shown, which was removed for clarification in other views. The ten labeled glomeruli are found in equivalent positions throughout stages and individuals. Note the variable number of horseshoe neuropils, i.e., three glomeruli in A0 $\left(\mathbf{C}^{\prime}\right)$. Nonglomerular neuropil in the AL forms a neuropilar bridge between AL and protocerebrum (arrow in $\mathbf{A}^{\prime \prime}$ ) or is found in the so called coarse neuropil (stars in $\mathbf{A}$ and $\left.\mathbf{A}^{\prime \prime}\right)$. Further clarification of glomerular 3D organization animations and inter active models of all these ALs are available online (http://online media.uni marburg.de/biologie/3d brain/). Bar $200 \mu \mathrm{m}$

On its posteroventral side, the AL neuropil loses its highly ordered glomerular organization and strays into the protocerebrum (Fig. 3A"). Some scattered synaptotagmin immunostaining not correlated to the glomerular neuropil was found in the so-called coarse neuropil of the central AL (Fig. 3a, A, A"; Dubuque et al. 2001).

\section{Reconstruction and identification of glomeruli}

We reconstructed 13 whole ALs. In addition to the sexspecific glomeruli of the MGC, we chose seven glomeruli that were most easily and reliably identified, based on their shape, volume, and location, in all specimens throughout the examined developmental stages (Figs. 2, 3). Four of them could be referred to by the nomenclature of Rospars and Hildebrand $(1992,2000)$. These were the largest of the "ordinary" glomeruli: G20 (light blue glomerulus in Figs. 2E, $\left.\mathrm{E}^{\prime}, \mathrm{F}, \mathrm{F}^{\prime}, 3\right)$, which lies posteromedial to the MGC (Rospars and Hildebrand 1992), the LPOG (yellow glomerulus in Figs. 2D, $\left.\mathrm{D}^{\prime}, \mathrm{E}, \mathrm{E}^{\prime}, 3\right)$, which corresponds to G64 and has been extensively described by Kent et al. (1986, 1999) and Guerenstein et al. (2004), and two anteriorly located glomeruli that we have named disc and discbase, which might correspond to G27/28 and G22/29 (dark red and green glomeruli, respectively, in Figs. 2A, $\mathrm{A}^{\prime}$,

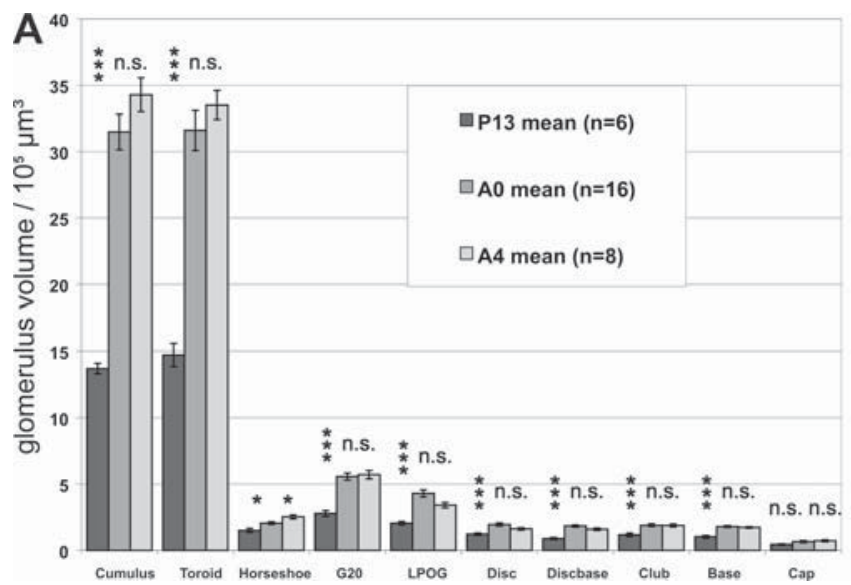

Fig. 4 Quantitative volume analysis of the three MGC glomeruli, the LPOG, and six "ordinary" glomeruli in P13 (dark, $n 6$ ALs) and adult animals (gray A0, $n 16$ ALs; light gray A4, $n 8$ ALs) of $M$. sexta (bars standard error, stars level of significance, $* * * P<0.001$,
B,B', 3). The discbase always appeared to be "U"-shaped in frontal sections (not shown). This glomerulus almost completely surrounded the disc, leaving only a small gap for the basal peduncle of the overlying disc.

The other three glomeruli were an anteroposteriorly elongated glomerulus that was found in the ventral AL just above the LPOG and that we named club (G60/58; light green glomerulus in Figs. 2D, $\left.\mathrm{D}^{\prime}, \mathrm{E}, \mathrm{E}^{\prime}, 3\right)$, and two ventromedian glomeruli that we called base (G61; blue glomerulus in Figs. 2D, $\left.\mathrm{D}^{\prime}, \mathrm{E}, \mathrm{E}^{\prime}, 3\right)$ and cap (G62; light orange glomerulus in Figs. 2E, $\mathrm{E}^{\prime}, \mathrm{F}, 3$ ), the latter exhibiting strong acetylcholine-esterase-like immunoreactivity (Homberg et al. 1995). Since the base and cap are closely associated, and since the cap lacks the typical proximal peduncle, they might have been considered as one glomerulus by Rospars and Hildebrand (1992, 2000).

In addition to the figures presented here, we have produced movies (.mpg files) of animated labeled slices and have reconstructed glomeruli for further clarification of the shape and organization in these ALs. We also provide interactive models (.vrml files) of reconstructed ALs (http://online-media.uni-marburg.de/biologie/3d brain/).

\section{Glomerulus volumes during pupal development}

We chose P13 as a representative pupal stage, since at this stage, all glomeruli of the adult animal are present and become distinguishable units (Tolbert et al. 1983). Nevertheless, the volumetric ratio at which these newly formed P13 and adult glomeruli were comparable was unclear. The glomeruli increased in volume between $40 \%$ and $130 \%$ from stages P13 to A0 (Fig. 4a), and the relative size increases of glomeruli as a percentage of the overall AL volume ranged from almost $20 \%$ to $90 \%$ (Fig. 4b). Exceptions were the cap glomerulus, which exhibited no significant size differences at all, and the horseshoe, whose significant growth $(P<0.05)$ was not mirrored in a relative size increase, adjusted to individual AL volumes (Fig. 4b).

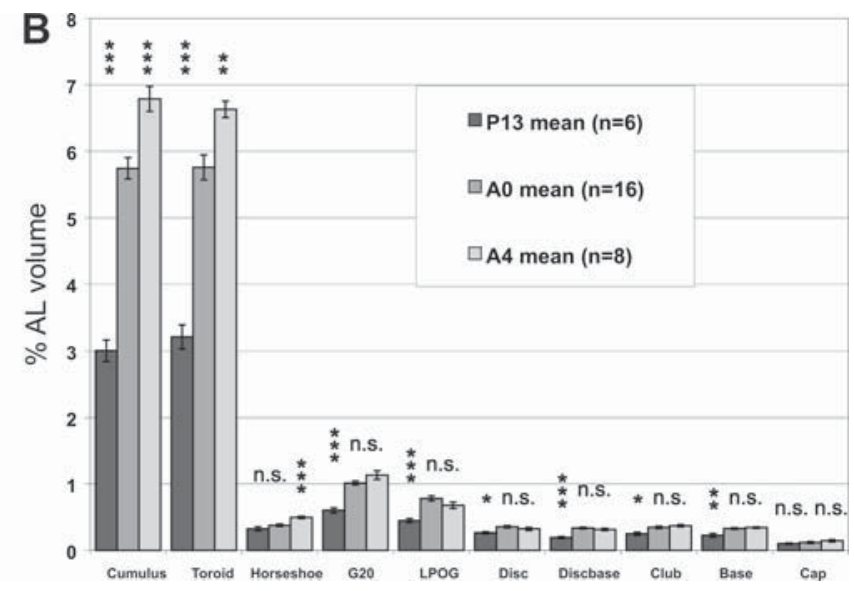

$* * P<0.01,{ }^{*} P<0.05$, n.s. not significant). a Mean values for absolute glomerular volumes. b Relative size of glomeruli as percentage of overall AL volume (glomerulus volume $\times 100 / \mathrm{AL}$ volume). 
Table 2 Complementing Fig. 4 this table shows the exact mean values and standard errors of (top) the glomerular and $\mathrm{AL}$ volumes $\left(\mu \mathrm{m}^{3}\right)$ and (bottom) the percentage of the glomerular volume compared with $\mathrm{AL}$ volume (glomerulus volume $\times 100 / \mathrm{AL}$ volume)

\begin{tabular}{lrllrlrrrrrr}
\hline Stage & Cumulus & Toroid & Horseshoe & G20 & LPOG & Disc & Discbase & Club & Base & Cap & Neurilemma \\
\hline P13 mean $(n$ 6) & $1,369,997$ & $1,472,315$ & 149,581 & 278,000 & 204,442 & 121,715 & 88,609 & 116,996 & 102,275 & 45,241 & $46,225,583$ \\
P13 standard error & 39,192 & 86,910 & 15,757 & 22,041 & 12,793 & 8,425 & 8,071 & 14,226 & 9,754 & 3,748 & $2,810,326$ \\
A0 mean ( $n$ 16) & $3,148,000$ & $3,159,776$ & 204,837 & 556,702 & 429,475 & 194,286 & 183,534 & 188,934 & 178,832 & 65,306 & $54,782,744$ \\
A0 standard error & 134,348 & 151,590 & 10,495 & 26,252 & 26,835 & 12,130 & 8,046 & 10,377 & 7,495 & 8,139 & $1,673,456$ \\
A4 mean $(n$ 8) & $3,429,388$ & $3,351,816$ & 252,591 & 570,906 & 340,824 & 162,077 & 158,752 & 187,322 & 172,660 & 71,703 & $50,644,375$ \\
A4 standard error & 127,317 & 110,138 & 15,357 & 32,064 & 20,060 & 9,044 & 9,127 & 11,190 & 5,442 & 7,917 & $1,850,346$ \\
& & & & & & & & & & & \\
& Cumulus & Toroid & Horseshoe & G20 & LPOG & Disc & Discbase & Club & Base & Cap \\
\hline P13 mean $(n$ 6) & 3.01 & 3.21 & 0.32 & 0.60 & 0.45 & 0.27 & 0.19 & 0.25 & 0.23 & 0.10 \\
P13 standard error & 0.16 & 0.18 & 0.03 & 0.04 & 0.03 & 0.01 & 0.01 & 0.02 & 0.03 & 0.01 \\
A0 mean $(n$ 16) & 5.74 & 5.76 & 0.38 & 1.01 & 0.78 & 0.36 & 0.34 & 0.35 & 0.33 & 0.12 \\
A0 standard error & 0.16 & 0.19 & 0.02 & 0.03 & 0.04 & 0.02 & 0.01 & 0.02 & 0.01 & 0.01 \\
A4 mean $(n$ 8) & 6.79 & 6.63 & 0.50 & 1.13 & 0.68 & 0.32 & 0.31 & 0.37 & 0.34 & 0.14 \\
A4 standard error & 0.19 & 0.12 & 0.02 & 0.07 & 0.05 & 0.02 & 0.02 & 0.02 & 0.01 & 0.02 \\
\hline
\end{tabular}

Interestingly, both the means of the cumulus and toroid almost doubled their volume $\left(1.37 \times 10^{6} \mathrm{\mu m}^{3}\right.$ and $1.47 \times 10^{6}$ $\mu \mathrm{m}^{3}$ at P13 compared with $3.15 \times 10^{6} \mu \mathrm{m}^{3}$ and $3.16 \times 10^{6}$ $\mu \mathrm{m}^{3}$, respectively, at $\mathrm{A} 0$ ) and thereby displayed the highest volume gains (Table 2). This was mirrored by the observation that approximately $27 \%$ of all glomerular volume in the P13 ALs compared with almost $30 \%$ in the A0 AL was allocated in the MGC. Thus, the relative percentage of pheromone-sensitive sensilla on the antennae (about one third) is reflected at the glomerulus volume level (Lee and Strausfeld 1990).

It is intriguing that the larger glomeruli exhibit higher growth rates than those of the smaller ones. This is not self-evident, since we have measured not surfaces but volumes and have compared relative size changes that are independent of the units involved (Fig. 4, Table 2). Within all P13 ALs, the size ranks of the corresponding glomeruli across individuals remain highly constant (coefficient of concordance $=0.93737, P<0.0001$ ), although there is no significant ranking between cumulus and toroid (coefficient of concordance $=0.44444 ; P<0.10248$ ).

\section{Glomerulus volumes during early adult development}

We found significant glomerulus size differences between adult moths differing by only 4 days in age. At A4, the means of the cumulus and toroid reached their highest values with $3.43 \times 10^{6} \mu \mathrm{m}^{3}$ and $3.35 \times 10^{6} \mu^{3}$, respectively, (Fig. 4a, Table 2); one third (33\%) of all neuropil volume in the ALs was now allocated in the MGC. Although the absolute volume differences were not significant between A0 and A4 (Fig. 4a), they became so for the MGC glomeruli after AL volume correction (Fig. 4b). All other glomeruli examined exhibited no significant differences between adult stages $\mathrm{A} 0$ and $\mathrm{A} 4$, neither between absolute (Fig. 4a) nor between corrected volumes (Fig. 4b). Nevertheless, within both adult stages examined, the size ranks of corresponding glomeruli across individuals remained highly constant (coefficient of concordance: A0 $=0.87992, P<0.0001 ; \mathrm{A} 4=0.94242, P<0.0001$ ). The cumulus and toroid, again, were still equally ranked (coefficient of concordance A $0=0.06250, P<0.31732$; $\mathrm{A} 4=0.0000, P<1.00000)$.

\section{Discussion}

Reliability of whole-mount immunostaining for 3D brain reconstructions

Dubuque et al. (2001) have demonstrated the suitability of the anti-synaptotagmin antibody for labeling neuropilar structures and that these structures represent glomeruli during AL development and in the adult AL. The combination of methanol/formaldehyde fixation and synaptotagmin antibody staining has allowed us clearly to label glomerular neuropil in M. sexta brain whole-mounts with almost a complete lack of an intensity gradient attributable to poor tissue permeation (Figs. 1, 2; Ott and Elphick 2003). We emphasize the quality of our labeling, since realistic reconstructions rely on good tissue permeation of the labels used, especially in whole-mounts. The employed fixation has allowed an almost homogeneous refractive index throughout the tissue (Gahm and Witte 1986); different refractive indices otherwise have a high impact on axial mismatch (Bucher et al. 2000). Because of the large size of the $\mathrm{AL}$ and the working distance of the objective, we have had to scan from two sides. This has had no obvious consequences on image stack quality (Fig. 1). Dehydration of tissue leads to shrinkage artifacts, as outlined by Bucher et al. (2000). However, by retaining the same protocol throughout all preparations, the volume differences measured between the glomeruli of individual animals at various stages should unequivocally reflect real differences and not artifacts. 
Usefulness of standard 3D glomeruli of the M. sexta AL

The usefulness of atlases and 3D databases in neuroscience is noncontentious (Ruffins et al. 2002; Van Essen 2002), and a plethora of 3D AL maps have arisen during the last few years (see Introduction). Computer-based 3D databases offer the opportunity to focus a variety of experimental approaches onto a standardized model system, even with respect to developmental events (Ruffins et al. 2002), whereas 3D atlases link these data to a common framework thereby compensating for individual differences (Van Essen 2002).

However, a comparable 3D map of the M. sexta AL has, to date, been missing. An existing map of the adult $M$. sexta AL, based on paraffin sections, provides an overview with regard to the absolute number, localization, and sex-specific dimorphisms of the glomeruli but does not provide 3D standard glomeruli (Rospars and Hildebrand 1992, 2000).

The M. sexta AL is a well-established model system for neurodevelopmental processes (for a review, see Tolbert et al. 2004). We have therefore provided not only a glomerular 3D standard for Manduca adults of various ages (A0, A4), but also for one stage (P13) during pupal development.

The formation of the glomeruli during pupal development can be divided into at least three phases. In a first phase including stages P5 to P7/8, the glomerular templates are formed by ORN axons and presumably by neurites of PNs (Malun et al. 1994; Oland and Tolbert 1996). In a second phase lasting from $P 7 / 8$ to about P12, the principal glomeruli are formed. This phase is emphasized in the AL by a distoproximally directed wave of synaptogenesis (Malun et al.1994). A third phase from P13 to adult eclosion includes growth and refinement of the glomerular neuropil (Dubuque et al. 2001). Our P13 standard glomeruli provide a tool for studying mechanisms involved in basic glomerular formation during phase 2. A0 standard glomeruli allow the study of mechanisms involved in the growth and refinement during phase 3.

We have only provided 3D standards for ten of the approximately 60 glomeruli. Our existing data set obtained with the CLSM in principal allows the production of a 3D standard for any glomerulus of the M. sexta AL. With this in mind, we suggest that customizable 3D standards should be produced in cases in which labeling efforts are focused on specific glomeruli that are needed in the relevant study. For the across-individual identification of corresponding glomeruli, multiple atlases outclass single atlases (Rohlfing et al. 2004). Our 30 reduced 3D AL maps might serve as such atlases and might lead to lesstime-consuming half-automated glomerulus identification. Some of these AL maps are available online in various forms, complementing the figures presented in this study; the others are available on request. In a phylogenetic context, the glomerular map presented in this work combined with existing atlases of other moths (Agrotis, Greiner et al. 2004; Heliothis and Helicoverpa, Berg et al. 2002; Spodoptera, Sadek et al. 2002) adds to a species- specific comparison of glomerular organization principles, as outlined by Greiner et al. (2004).

Comparison with existing data concerning the $M$. sexta AL

The first extrapolated estimates of overall glomerular volume during development of $M$. sexta ALs produced higher values (Tolbert 1989) than we have measured. This difference may be based on systematic differences in quantification techniques and on individual size differences between the animals used. However, what are the reasons for the glomerular volume increases? Mechanisms involved in volume increases are certainly different during development and in the adult but might share common principles depending on the stage of development.

Cell proliferation in the AL has finished at P3 (Hildebrand et al. 1997), and programmed neuronal cell death does not occur in the developing AL of $M$. sexta (Schachtner et al. 2004a). Moreover, glial cells, which significantly contribute to glomerulus formation during phase 2 of pupal AL development, presumably do not participate in glomerulus maturation and growth during later stages (Baumann et al. 1996; Oland et al. 1999; for a review, see Tolbert et al. 2004). This leaves anatomical changes in the neuronal components of the AL during phase 3 and in the adult as the basis for volume changes in the glomeruli.

These anatomical changes can principally result from three mechanisms: (1) changes in the branching pattern of neurites, (2) the swelling or shrinking of neurites, and (3) synapse formation or decomposition. (1) At the lightmicroscopical level, neurite elaboration in the AL of $M$. sexta appears to be finished by about P12 (Tolbert et al. 1983; Oland et al. 1990). We know of no information for $M$. sexta as to whether any of the neurons involved in glomerular composition change their branching pattern during phase 3 or in the adult. In honeybees, neuritic arbors in glomeruli undergo continuous maturation of their branching pattern during pupal and early adult AL development; this includes the pruning of dendritic arbors in a subset of LNs, but an effect of pruning on glomerulus volume has not been examined (Devaud and Masson 1999). (2) Tolbert (1989) has found, in an in-depth electron-microscopic study of the developing $M$. sexta AL, increasing diameters of glomerular processes during development, although the various cell types could not be resolved. Between P4 to P7, Tolbert described glomerular neuritic processes with diameters of $0.1-0.5 \mu \mathrm{m}$ increasing, at P17, to a mean diameter of $0.2-1.0 \mu \mathrm{m}$, and finally reaching $2 \mu \mathrm{m}$ in diameter at adult eclosion (Tolbert 1989). (3) The formation of new synaptic junctions seems to stall around stage P12 (Tolbert et al. 1983). These data are supported by a recent study from Dubuque et al. (2001) who have found the highest synaptotagmin immunostaining in P12 glomeruli. After P12, synaptotagmin density decreases, and the glomeruli grow in size (Dubuque et al. 2001). In vertebrates, similar results have been ob- 
tained, supporting the view of a main phase of synaptogenesis followed by a second phase of maturation and refinement (Yuste and Bonhoeffer 2004). Thus, the net number of synapses might only contribute to volume changes to a small extent, but no studies are available that examine the correlation of the numbers of synapses with neuropilar size during development.

\section{Correlates of adult neuronal plasticity}

Data regarding neuronal plasticity in adult $M$. sexta is scarce. Daly et al. (2004) have recently shown that olfactory conditioning is combined with a restructuring of electrical odor response patterns in the AL. Work on adult neuronal plasticity in the honeybee has demonstrated that increases in adult glomerular volume are activity-dependent and correlated with better learning performance (Winnington et al. 1996; Sigg et al. 1997). Quantitative electron-microscopic studies have lead to the suggestion that volume increase and synapse number changes are independent processes that both contribute to structural plasticity in the AL, although synapse reorganization might only play a minor role on neuropilar volume (Brown et al. 2002). Age effects on bee mushroom body volumes must be distinguished from experience effects; the first 5 days of adult brain development seem to be functionally separated from later maturation effects (Farris et al. 2001); this is supported by synapse counts in certain glomeruli (Brown et al. 2004).

Devaud et al. (2003) also argue in this vein, as they have found a rapid increase in glomerulus size during late pupal development and ongoing heterogeneous glomerulus growth in young adults in Drosophila. This adult volume gain can be induced up to a critical age by specific odors and, unlike pupal growth, seems to involve a cAMPdependent signalling pathway (Devaud et al. 2001). In Agrotis ipsilon pheromone-specific odor processing seems to be dependent upon juvenile hormone and age (Anton and Gadenne 1999; Gadenne and Anton 2000; Greiner et al. 2002). Two main possibilities may explain the induction of adult neuropilar growth: first, it might represent the final maturation processes, which are possibly hormonally controlled, or second, it might be a manifestation of early adult neural plasticity depending on sensory input (as discussed in Julian and Gronenberg 2002). A separation of these two possibilities may not be possible; indeed, they may build upon each other (Devaud et al. 2003).

We have observed the post-eclosional growth of sexspecific glomeruli. After eclosion, the animals are confronted with female pheromone for the first time. Thus, the neuronal activity of pheromone-specific olfactory receptor neurons might account for this early adult glomerular plasticity. For $M$. sexta, recent results further suggest the occurrence of a sensitive phase during early adult AL development. The reactive oxygen species scavenger $\mathrm{Cu} / \mathrm{Zn}$-superoxide dismutase (SOD) is present in a subset of AL neurons from A0 to A2/3 (Schachtner et al. 2004a). Schachtner et al. (2004a) discuss SOD as a cellular marker unmasking cells undergoing their last refinements to cope with the odor information provided from the olfactory receptor neurons. Furthermore, pheromone-specific MGC-ORNs maintain certain glycosylation patterns into adulthood (Gibson et al. 2004). At the behavioral level, adult female $M$. sexta exhibit changing responses to host plant volatiles, depending on age and mating status (Mechaber et al. 2002). The underlying principle might be comparable to that in males, since the female glomeruli specialized to host plant odors correspond to the MGC in males (Rospars and Hildebrand 2000).

Regarding the question as to which cell types contribute to adult volume plasticity, genetic work of Scott et al. (2003a,b) on the Drosophila visual system exclude secondorder interneurons; if extrapolated to the olfactory system of M. sexta, this could mean that LNs and ORNs, but not PNs, are primarily responsible for glomerular volume differences. Of course, it has to be kept in mind that functional odor processing is not dependent on glomerular structure: if normal glomerular development is prevented, the animals are still competent to process odors (Oland and Tolbert 1988; Oland et al. 1988). Additionally, Willis et al. (1995) have demonstrated the odor-modulated flight of female moths lacking glomerular neuropil organization in the AL.

With respect to the questions that we initially raised, we have found (1) a tremendous increase in size in ten identified glomeruli during the last third of pupal development. These ten glomeruli are reliably identifiable in different individuals, and their volume gain has been quantified, thereby supplying (2) an anatomical and volumetric standard for future studies during development and in adults. Based on these glomerular standards, which are available online, we are now able to address issues of neuropilar development, such as the contribution of different cell types to size increase, or to investigate the influences of neuroactive compounds and electrical activity on neuropil growth in defined glomeruli.

Acknowledgements The authors thank Dr. Kaushiki Menon (Caltech, USA) for kindly providing the synaptotagmin antiserum and Dr. Robert Brandt (Zuse Institute, Berlin, Germany) for his excellent support with respect to the AMIRA software. This study was supported by a grant from the Deutsche Forschungsge meinschaft (Grant Scha 678/3 3) to JS.

\section{References}

Anton S, Gadenne C (1999) Effect of juvenile hormone on the central nervous processing of sex pheromone in an insect. Proc Natl Acad Sci USA 96:5764 5767

Baumann PM, Oland LA, Tolbert LP (1996) Glial cells stabilize axonal protoglomeruli in the developing olfactory lobe of the moth Manduca sexta. J Comp Neurol 373:118 128

Bell RA, Joachim FA (1978) Techniques for rearing laboratory colonies of the tobacco hornworm, Manduca sexta and pink ballworms. Ann Entomol Soc Am 69:365 373 
Berg BG, Galizia CG, Brandt R, Mustaparta H (2002) Digital atlases of the antennal lobe in two species of tobacco budworm moths, the Oriental Helicoverpa assulta (male) and the American Heliothis virescens (male and female). J Comp Neurol 446 123134

Brown SM, Napper RM, Thompson CM, Mercer AR (2002) Stereological analysis reveals striking differences in the struc tural plasticity of two readily identifiable glomeruli in the antennal lobes of the adult worker honeybee. J Neurosci 22:8514 8522

Brown SM, Napper RM, Mercer AR (2004) Foraging experience, glomerulus volume, and synapse number: a stereological study of the honey bee antennal lobe. J Neurobiol 60:40 50

Bucher D, Scholz M, Stetter M, Obermayer K, Pflüger HJ (2000) Correction methods for three dimensional reconstructions from confocal images. I. Tissue shrinking and axial scaling. J Neurosci Methods 100:135 143

Chambille I, Rospars JP (1981) Le deutocérébron de la blatte Blaberus craniifer Burm. (Dictyoptera: Blaberidae). Etude qualitative et identification visuelle de glomérules. Int J Insect Morphol Embryol 10:141 165

Chiang AS, Liu YC, Chiu SL, Hu SH, Huang CY, Hsieh CH (2001) Three dimensional mapping of brain neuropils in the cock roach, Diploptera punctata. J Comp Neurol 440:1 11

Daly KC, Christensen TA, Lei H, Smith BH, Hildebrand JG (2004) Learning modulates the ensemble representations for odors in primary olfactory networks. Proc Natl Acad Sci USA 101: 1047610481

Devaud JM, Masson C (1999) Dendritic pattern development of the honeybee antennal lobe neurons: a laser scanning confocal microscopic study. J Neurobiol 39:461 474

Devaud JM, Acebes A, Ferrus A (2001) Odor exposure causes central adaptation and morphological changes in selected olfactory glomeruli in Drosophila. J Neurosci 21:6274 6282

Devaud JM, Acebes A, Ramaswami M, Ferrus A (2003) Structural and functional changes in the olfactory pathway of adult Drosophila take place at a critical age. J Neurobiol 56:13 23

Dubuque SH, Schachtner J, Nighorn AJ, Menon KP, Zinn K, Tolbert LP (2001) Immunolocalization of synaptotagmin for the study of synapses in the developing antennal lobe of Manduca sexta. J Comp Neurol 441:277 287

Eisthen HL (2002) Why are olfactory systems of different animals so similar? Brain Behav Evol 59:273 293

Farris SM, Robinson GE, Fahrbach SE (2001) Experience and age related outgrowth of intrinsic neurons in the mushroom bodies of the adult worker honeybee. J Neurosci 21:6395 6404

Flanagan D, Mercer AR (1989) An atlas and 3 D reconstruction of the antennal lobes in the worker honey bee, Apis mellifera $\mathrm{L}$. (Hymenoptera: Apidae). Int J Insect Morphol Embryol 18:145 159

Gadenne C, Anton S (2000) Central processing of sex pheromone stimuli is differentially regulated by juvenile hormone in a male moth. J Insect Physiol 46:1195 1206

Gahm T, Witte S (1986) Measurement of the optical thickness of transparent tissue layers. J Microsc 141:101 110

Galizia CG, McIlwrath SL, Menzel R (1999) A digital three dimensional atlas of the honeybee antennal lobe based on optical sections acquired by confocal microscopy. Cell Tissue Res 295:383 394

Gibson NJ, Hildebrand JG, Tolbert LP (2004) Glycosylation patterns are sexually dimorphic throughout development of the olfactory system in Manduca sexta. J Comp Neurol 476:1 18

Greiner B, Gadenne C, Anton S (2002) Central processing of plant volatiles in Agrotis ipsilon males is age independent in contrast to sex pheromone processing. Chem Senses 27:45 48

Greiner B, Gadenne C, Anton S (2004) Three dimensional antennal lobe atlas of the male moth, Agrotis ipsilon: a tool to study structure function correlation. J Comp Neurol 475:202 210

Guerenstein PG, Christensen TA, Hildebrand JG (2004) Sensory processing of ambient $\mathrm{CO}_{2}$ information in the brain of the moth Manduca sexta. J Comp Physiol [A] 190:707 725
Haddad D, Schaupp F, Brandt R, Manz G, Menzel R, Haase A (2004) NMR imaging of the honeybee brain. J Insect Sci 4:1 7

Hildebrand JG, Shepherd GM (1997) Mechanisms of olfactory discrimination: converging evidence for common principles across phyla. Annu Rev Neurosci 20:595 631

Hildebrand JG, Rössler W, Tolbert LP (1997) Postembryonic development of the olfactory system in the moth Manduca sexta: primary afferent control of glomerular development. Cell Dev Biol 8:163 170

Homberg U, Hoskins SG, Hildebrand JG (1995) Distribution of acetylcholinesterase activity in the deutocerebrum of the sphinx moth Manduca sexta. Cell Tissue Res 279:249 259

Huetteroth W, Schachtner J (2003) 3D reconstructions of pupal and adult glomeruli in the antennal lobe of the sphinx moth Manduca sexta. Proc Fifth German Neurosci Soc Conf 689:735

Jindra M, Huang JY, Malone F, Asahina M, Riddiford LM (1997) Identification and mRNA developmental profiles of two ultra spiracle isoforms in the epidermis and wings of Manduca sexta. Insect Mol Biol 6:41 53

Julian GE, Gronenberg W (2002) Reduction of brain volume cor relates with behavioral changes in queen ants. Brain Behav Evol 60:152 164

Kent KS, Harrow ID, Quartararo P, Hildebrand JG (1986) An accessory olfactory pathway in Lepidoptera: the labial pit organ and its central projections in Manduca sexta and certain other sphinx moths and silk moths. Cell Tissue Res 245:237 245

Kent KS, Oland LA, Hildebrand JG (1999) Development of the labial pit organ glomerulus in the antennal lobe of the moth Manduca sexta: the role of afferent projections in the formation of identifiable olfactory glomeruli. J Neurobiol 40:28 44

Laissue PP, Reiter C, Hiesinger PR, Halter S, Fischbach KF, Stocker RF (1999) Three dimensional reconstruction of the antennal lobe in Drosophila melanogaster. J Comp Neurol 405:543 552

Lee JK, Strausfeld NJ (1990) Structure, distribution and number of surface sensilla and their receptor cells on the olfactory appendage of the male moth Manduca sexta. J Neurocytol 19:519 538

Malun D, Oland LA, Tolbert LP (1994) Uniglomerular projection neurons participate in early development of olfactory glomeruli in the moth Manduca sexta. J Comp Neurol 350:1 22

Mechaber WL, Capaldo CT, Hildebrand JG (2002) Behavioral responses of adult female tobacco hornworms, Manduca sexta, to hostplant volatiles change with age and mating status. J Insect Sci 2:1 8

Oland LA, Tolbert LP (1988) Effects of hydroxyurea parallel the effects of radiation in developing olfactory glomeruli in insects. J Comp Neurol 278:377 387

Oland LA, Tolbert LP (1996) Multiple factors shape development of olfactory glomeruli: insights from an insect model system. J Neurobiol 30:92 109

Oland LA, Tolbert LP, Mossman KL (1988) Radiation induced reduction of the glial population during development disrupts the formation of olfactory glomeruli in an insect. J Neurosci 8:353 367

Oland LA, Orr G, Tolbert LP (1990) Construction of a proto glomerular template by olfactory axons initiates the formation of olfactory glomeruli in the insect brain. J Neurosci 10:2096 2112

Oland LA, Marrero HG, Burger I (1999) Glial cells in the develop ing and adult olfactory lobe of the moth Manduca sexta. Cell Tissue Res 297:527 545

Ott SR, Elphick MR (2003) New techniques for whole mount NADPH diaphorase histochemistry demonstrated in insect gan glia. J Histochem Cytochem 51:523 532

Rein K, Zöckler M, Heisenberg M (1999) A quantitative three dimensional model of the Drosophila optic lobes. Curr Biol 9:93 96

Rein K, Zöckler M, Mader MT, Grubel C, Heisenberg M (2002) The Drosophila standard brain. Curr Biol 12:227 231 
Rohlfing T, Brandt R, Menzel R, Maurer CR Jr (2004) Evaluation of atlas selection strategies for atlas based image segmentation with application to confocal microscopy images of bee brains. Neuroimage 21:1428 1442

Rospars JP (1983) Invariance and sex specific variations of the glomerular organization in the antennal lobes of a moth, Mamestra brassicae, and a butterfly, Pieris brassicae. J Comp Neurol 220:80 96

Rospars JP, Hildebrand JG (1992) Anatomical identification of glomeruli in the antennal lobes of the male sphinx moth Manduca sexta. Cell Tissue Res 270:205 227

Rospars JP, Hildebrand JG (2000) Sexually dimorphic and isomor phic glomeruli in the antennal lobes of the sphinx moth Manduca sexta. Chem Senses 25:119 129

Ruffins SW, Jacobs RE, Fraser SE (2002) Towards a Tralfamadorian view of the embryo: multidimensional imaging of development. Curr Opin Neurobiol 12:580 586

Sadek MM, Hansson BS, Rospars JP, Anton S (2002) Glomerular representation of plant volatiles and sex pheromone compo nents in the antennal lobe of the female Spodoptera littoralis. J Exp Biol 205:1363 1376

Schachtner J, Huetteroth W, Nighorn A, Honegger HW (2004a) Copper/zinc superoxide dismutase like immunoreactivity in the metamorphosing brain of the sphinx moth Manduca sexta. J Comp Neurol 469:141 152

Schachtner J, Trosowski B, D'Hanis W, Stubner S, Homberg U (2004) Development and steroid regulation of RFamide immunoreactivity in antennal lobe neurons of the sphinx moth Manduca sexta. J Exp Biol 207:2389 2400

Schwartz LM, Truman JW (1983) Hormonal control of rates of metamorphic development in the tobacco hornworm Manduca sexta. Dev Biol 99:103 114

Scott EK, Reuter JE, Luo L (2003a) Dendritic development of Drosophila high order visual system neurons is independent of sensory experience. BMC Neurosci 4:14

Scott EK, Reuter JE, Luo L (2003b) Small GTPase Cdc42 is required for multiple aspects of dendritic morphogenesis. J Neurosci 23:3118 3123

Sigg D, Thompson CM, Mercer AR (1997) Activity dependent changes to the brain and behavior of the honey bee, Apis mellifera (L.). J Neurosci 17:7148 7156
Smid HM, Bleeker MA, Loon JJ van, Vet LE (2003) Three dimensional organization of the glomeruli in the antennal lobe of the parasitoid wasps Cotesia glomerata and C. rubecula. Cell Tissue Res 312:237 248

Strausfeld NJ, Hildebrand JG (1999) Olfactory systems: common design, uncommon origins? Curr Opin Neurobiol 9:634 639

Tolbert LP (1989) Afferent axons from the antenna influence the number and placement of intrinsic synapses in the antennal lobes of Manduca sexta. Synapse 3:83 95

Tolbert LP, Sirianni PA (1990) Requirement for olfactory axons in the induction and stabilization of olfactory glomeruli in an insect. J Comp Neurol 298:69 82

Tolbert LP, Matsumoto SG, Hildebrand JG (1983) Development of synapses in the antennal lobes of the moth Manduca sexta during metamorphosis. J Neurosci 3:1158 1175

Tolbert LP, Oland LA, Tucker ES, Gibson NJ, Higgins MR, Lipscomb BW (2004) Bidirectional influences between neurons and glial cells in the developing olfactory system. Prog Neurobiol 73:73 105

Truman JW (1996) Metamorphosis of the insect nervous system. In: Gilbert LI (ed) Metamorphosis: postembryonic reprogramming of gene expression in amphibian and insect cells. Academic Press, Orlando, pp 283320

Van Essen DC (2002) Windows on the brain: the emerging role of atlases and databases in neuroscience. Curr Opin Neurobiol $12: 574579$

Weevers RD (1966) A lepidopteran saline: effects of inorganic cation concentrations on sensory, reflex and motor responses in a herbivorous insect. J Exp Biol 44:163 175

Willis MA, Butler MA, Tolbert LP (1995) Normal glomerular organization of the antennal lobes is not necessary for odor modulated flight in female moths. J Comp Physiol [A] 176:205 216

Winnington AP, Napper RM, Mercer AR (1996) Structural plasticity of identified glomeruli in the antennal lobes of the adult worker honey bee. J Comp Neurol 365:479 490

Yuste R, Bonhoeffer T (2004) Genesis of dendritic spines: insights from ultrastructural and imaging studies. Nat Rev Neurosci 5:24 34

Zöckler M, Rein K, Brandt R, Stalling D, Hege H (2001) Creating virtual insect brains with AMIRA. ZIB Report $0132: 1 \quad 11$ 